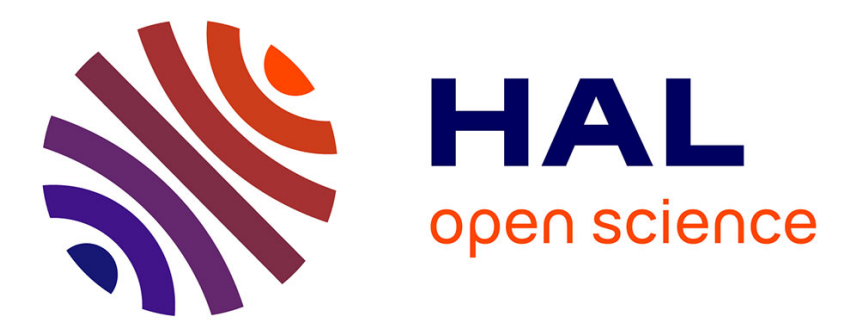

\title{
Characterization of inhomogeneous weakly absorbing thin films by photothermal deflection spectroscopy, applications to hydrogenated amorphous silicon films
}

L. Chahed, J. Frigerio, F. Jérémie, M. Thèye

\section{- To cite this version:}

L. Chahed, J. Frigerio, F. Jérémie, M. Thèye. Characterization of inhomogeneous weakly absorbing thin films by photothermal deflection spectroscopy, applications to hydrogenated amorphous silicon films. Journal de Physique IV Proceedings, 1994, 04 (C7), pp.C7-121-C7-124. 10.1051/jp4:1994729 . jpa-00253257

\section{HAL Id: jpa-00253257 https://hal.science/jpa-00253257}

Submitted on 1 Jan 1994

HAL is a multi-disciplinary open access archive for the deposit and dissemination of scientific research documents, whether they are published or not. The documents may come from teaching and research institutions in France or abroad, or from public or private research centers.
L'archive ouverte pluridisciplinaire HAL, est destinée au dépôt et à la diffusion de documents scientifiques de niveau recherche, publiés ou non, émanant des établissements d'enseignement et de recherche français ou étrangers, des laboratoires publics ou privés. 


\title{
Characterization of inhomogeneous weakly absorbing thin films by photothermal deflection spectroscopy, applications to hydrogenated amorphous silicon films
}

\author{
L. Chahed*, J.M. Frigerio, F. Jérémie and M.L. Thèye
}

Laboratoire d'Optique des Solides, URA 781 du CNRS, Université Pierre et Marie Curie, Case 80, 4 place Jussieu, 75252 Paris cedex 05, France

* Laboratoire d'Optique des Couches Minces, Unité de Recherche Physique, Université d'Oran es-Sénia, Oran, Algérie

\begin{abstract}
We explore the possibilities offered by transverse photothermal deflection spectroscopy at low modulation frequencies for studying variations of the optical absorption coefficient of hydrogenated amorphous silicon thin films across their thickness, with a special emphasis on the low energy range.
\end{abstract}

\section{INTRODUCTION}

Sensitive spectroscopic techniques based on photothermal effects, i.e. photothermal deflection spectroscopy (PDS) and, to a lesser extent, photoacoustic spectroscopy (PAS), are presently widely used to determine the optical absorption coefficient spectra $\alpha(\hbar \omega)$ of hydrogenated amorphous silicon (a-Si:H) and related materials thin films in their low absorption range. For energies smaller than the optical gap, $\alpha(\hbar \omega)$, which in amorphous semiconductors is simply given by a convolution of the densities of initial $\left(\mathrm{N}_{\mathrm{v}}\right)$ and final $\left(\mathrm{N}_{\mathrm{c}}\right)$ states separated in energy by $\hbar \omega$ :

$$
\alpha(\hbar \omega)=\frac{K}{\hbar \omega} \int N_{v}(E) \cdot N_{c}(E+\hbar \omega) d E,
$$

is due to optical transitions involving the localized electronic states introduced into the gap by the disorder (band tails) and the defects - mainly dangling bonds (deeper states). Very useful information about the density and the energy distribution of these localized states can thus be deduced from the $\alpha(\hbar \omega)$ spectra by using appropriate models for the density of states (DOS) in the (pseudo) gap. The measurements are usually performed at low modulation frequencies, i.e. under conditions where the films are thermally thin. The modulus of the PDS (PAS) signal is then simply proportional to the film absorptance [1] :

$$
|\mathrm{S}(\hbar \omega)|=\mathrm{S}_{\mathrm{o}} \mathrm{A}(\hbar \omega)=\mathrm{S}_{\mathrm{o}}[1-(\mathrm{R}(\hbar \omega)+\mathrm{T}(\hbar \omega))]
$$

where $R$ and $T$ are the film reflectance and transmittance, and $S_{O}$ is a constant including the thermal and geometrical parameters. Since $\alpha$ increases with $\hbar \omega$ by several orders of magnitude in the considered spectral range (absorption edge), the calibration of the $|S(\hbar \omega)|$ spectra can easily be done by fitting to the A $(\hbar \omega)$ spectrum computed with the optical constants deduced from transmission measurements in the high energy / high absorption range. When $\alpha \mathrm{d}<0.1$, the $\mathrm{A}(\hbar \omega)$ thin film expressions become [2] :

$A_{c}=\frac{\left(1-r_{o}^{2}\right)\left[1-r_{1}^{2} e^{-2 \alpha d}-\left(1-r_{1}^{2}\right) e^{-\alpha d}\right]}{1+r_{o}^{2} r_{1}^{2} e^{-2 \alpha d}+2 r_{o} r_{1} e^{-\alpha d} \cos (2 n \beta)} ; A_{I}=\frac{\left(1-r_{o}^{2}\right)\left[1-r_{1}^{2} e^{-2 \alpha d}-\left(1-r_{1}^{2}\right) e^{-\alpha d}\right]}{1-r_{o}^{2} r_{1}^{2} e^{-2 \alpha d}}$

for coherent $\left(\mathrm{A}_{\mathrm{C}}\right)$ and incoherent $\left(\mathrm{A}_{\mathrm{I}}\right)$ multiple reflections inside the film; $r_{0}$ and $r_{1}$ are the (real) Fresnel reflection coefficients at the surface and the interface and depend only on the refractive index of the different media, and $\beta=2 \pi \mathrm{d} / \lambda . A_{c}(\hbar \omega)$ presents interference fringes [3], which are currently observed on the PDS (PAS) spectra. In order to determine $\alpha(\hbar \omega)$, one takes advantage of the relation : $\mathrm{A}_{\mathrm{I}}=\left(\mathrm{A}_{\mathrm{c}}^{\mathrm{Max}} \cdot \mathrm{A}_{\mathrm{c}}^{\min }\right)^{1 / 2} \simeq \alpha \mathrm{d}$, so that $\alpha \mathrm{d}(\hbar \omega)$ is simply obtained by taking the geometric average of the fringes in the calibrated $|S(\hbar \omega)|$ spectra. 
The analysis of the PDS (PAS) data by the procedure described above may not however yield the correct $\alpha(\hbar \omega)$ spectrum. On the one hand, the film is assumed to be optically homogeneous ( $\mathrm{n}$ and $\alpha$ constant across the film thickness). The observation that $\mathrm{A}(\hbar \omega) \approx \alpha(\hbar \omega)$.d does not scale with thickness in the low absorption range suggests the presence of more absorbing (i.e. more defective) surface and/or interface layers, responsible for an increase of the measured "average" $\alpha(\hbar \omega)$ with respect to the true "bulk" one $[4,5]$. It is therefore important to be able to detect such non-homogeneities (which do not show up in the $T(\hbar \omega)$ spectra), and to characterize them, in order to reach the bulk DOS. On the other hand, numerical simulations of $\alpha(\hbar \omega)$ using realistic models for the DOS (exponential band tails and Gaussian defect band) [6] show that the $\alpha$ values should drop rapidly at low energies since the density of available states for optical transitions on either side of the Fermi level decreases by several orders of magnitude for small $\hbar \omega$ values. While the $\alpha(\hbar \omega)$ spectra derived from photoconductivity (CPM) measurements commonly exhibit such a behaviour, the PDS-derived ones usually remain rather flat down to quite low energies, indicating that there is some additional absorption in the low energy range [6]. In the present paper we will consider these two points, which are indeed very important both for material characterization and for the understanding of the relation between disorder and defects and growth mechanisms.

\section{EXPERIMENT AND THEORETICAL MODELS.}

The samples were undoped a-Si:H films deposited on glass or silica substrates by glow discharge decomposition of silane under different plasma conditions and at different substrate temperatures, in two different reactors. The transverse PDS measurements were performed in $\mathrm{CCl}_{4}$ at a $13 \mathrm{~Hz}$ modulation frequency. We used a compact mirage cell [7] which could be rotated by $180^{\circ}$ with respect to the pump beam without changing the sample/probe beam configuration. The PDS spectra were carefully calibrated as explained above. All the numerical simulations were performed with exact thin film expressions for $\mathrm{R}$ and $\mathrm{T}$, using a matrix formalism well adapted to multilayer systems [8]. Multiple reflections inside the substrate were neglected. $\alpha$ and/or $\mathrm{n}$ gradients were obtained by dividing the film into a stack of several layers with appropriate thickness and optical constants.

\section{RESULTS AND DISCUSSION.}

A clever method has been proposed to detect the presence of thin surface/interface absorbing layers and to determine their location [9]. It consists in comparing the PDS spectra obtained with front $(F)$ and rear (R) illumination, the detection configuration remaining identical. The fringe contrast $A_{c}^{M a x} / A_{c}^{\min }$ of these spectra in the low absorption range is indeed strongly enhanced for $F(R)$ illumination in the presence of a thin surface (interface) film. Many studies $[9,10,11]$ have explored the possibilities and limits of this method, and the relation between the observed contrast effects and the ratio between the surface layer thickness $d_{\mathbf{S}}$ and the wavelength of the standing waves inside the film has been clearly established [11]. The contrast effects expected when the surface layer thickness increases or when $\alpha$ varies continuously over the film thickness, as well as the simultaneous changes in the average absorption level are illustrated in figure $1 \mathrm{a}$ and $\mathrm{lb}$. As $\mathrm{d}_{\mathrm{S}}$ increases, the fringe contrast decreases but the film non-homogeneity can still be detected through the deformation of the fringe system, which is not the same for $F$ and $R$ illumination. When $d_{S}$ increases further, or when the $\alpha$ gradient is linear, the $F$ and $R$ fringe systems become very close. According to the contrast criterion, the films look homogeneous but their average $\alpha(\hbar \omega)$ are much higher than the "bulk" value. When $\alpha$ decreases rapidly from its surface value to its bulk value over a small depth, the effects are similar to those of a homogeneous "average" surface layer with appropriate $\alpha_{\mathrm{S}}$ and $\mathrm{d}_{\mathrm{s}}$. The $\mathrm{n}$ variations which are likely to accompany the $\alpha$ variations have little influence on the fringe contrast and the average absorption level. On the contrary, they introduce a modulation of the $T(\hbar \omega)$ minima values, while $\alpha$ variations alone do not affect the $\mathrm{T}(\hbar \omega)$ spectra in a detectable way. Figure $1 \mathrm{c}$ shows as an example the calibrated F and R PDS-derived A ( $\hbar \omega)$ spectra for an undoped a-Si:H film $(d=1.15 \mu \mathrm{m})$ deposited from silane diluted in Helium $(40 \%)$ at high pressure (550 mTorr) and high r.f. power (15 W), which results in a high deposition rate $(9 \AA / \mathrm{s})$. Under such plasma conditions the substrate temperature $\left(200^{\circ} \mathrm{C}\right)$ was too low to permit the growing film to "equilibrate" during deposition [12], so that the amount of disorder and the density of defects are expected to increase from the interface to the surface. This is confirmed in figure $1 \mathrm{c}$, where the fringe contrast is much larger in the $\mathrm{F}$ spectrum. The fact that the 

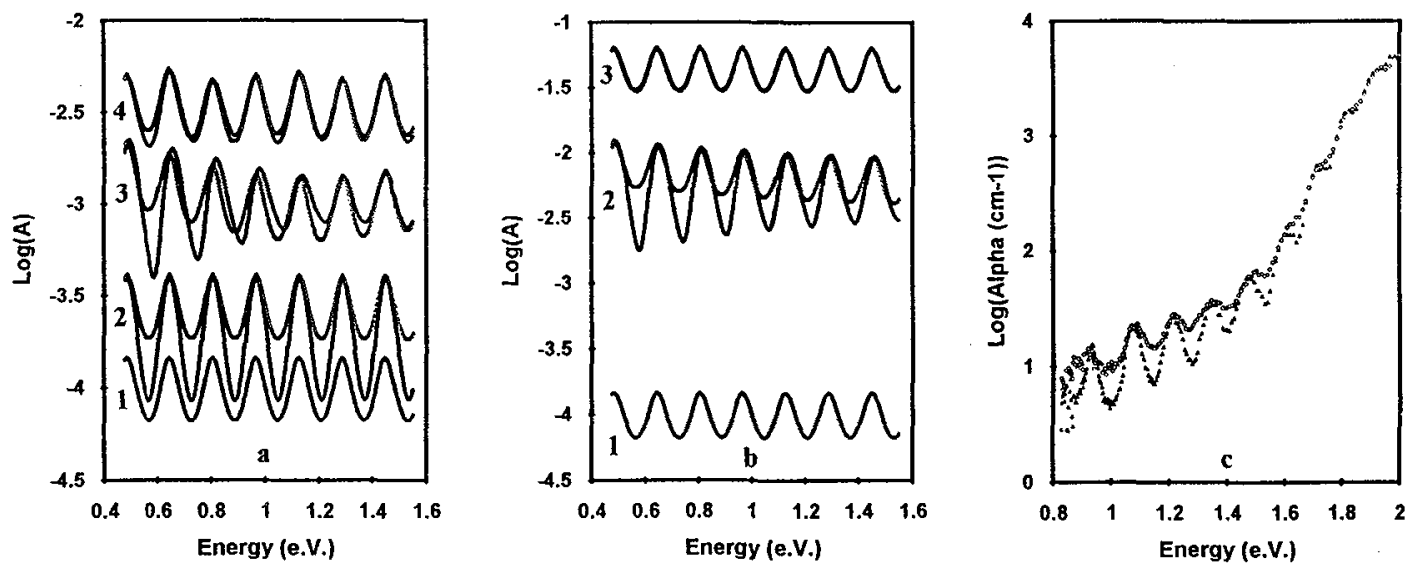

Figure 1 : Computed absorptance spectra for a homogeneous film ( $\left.n=3.85, \alpha=1 \mathrm{~cm}^{-1}, d=1 \mu \mathrm{m}\right)$ (I) and for a similar film (total $d=1 \mu \mathrm{m}$ ) including surface layers of increasing thickness : $n_{S}=3.85, \alpha_{S}=$ $10^{3} \mathrm{~cm}^{-1}, d_{s}=10$ (2), 100 (3), 333 (4) nm (a) or presenting an a gradient from $10^{3} \mathrm{~cm}^{-1}$ (surface) to 1 $\mathrm{cm}^{-1}$ (interface) : linear (2) and exponential (3) (b), for front ( $\rightarrow$ ) and rear ( $\Delta$ ) illumination ; experimental $\alpha(\hbar \omega)$ spectra obtained from PDS data with front ( ) and rear (o) illumination for a nonhomogeneous a-Si:H film $(d=1.15 \mu \mathrm{m})$ (c).

contrast increases towards low energies suggests that the surface defective material extends rather deep into the film. The difference in the fringe contrast observed in the exponential part of the edge indicates that the defective part has probably also a larger gap, i.e. more bonded hydrogen.

We now turn to the lowest energy part of the PDS spectra. As stressed before, the $\alpha$ values do not follow the behaviour predicted from expression (1) (contrary to the CPM spectra). Differences must of course exist between the PDS and CPM - derived $\alpha(\hbar \omega)$ spectra at room temperature, since PDS probes all possible optical transitions at $\hbar \omega$, while CPM selects those which give mobile electrons, i.e. for which the final states lie above the conduction band mobility edge and just below it in the conduction band tail [6]. However, they should essentially show up around $1.3 \mathrm{eV}$. Slight differences should also occur at very low energies, but are unable to explain the "flatness" of the PDS spectra. This flatness has often be ascribed to additional absorption coming from the substrate. Such an interpretation can easily be verified experimentally, because absorption in the substrate (which is thermally thick) results in a change in the phase of the PDS signal, which should on the contrary remain constant, and identical for $F$ and $R$ illumination, if absorption is coming from the film only. Such a change was observed in some cases $[11,13]$ Figure 2a shows a comparison of the PDS-derived $\alpha(\hbar \omega)$ spectra with F and R illumination, for a devicequality a-Si:H film $(\mathrm{d}=1 \mu \mathrm{m})$ deposited on an $\mathrm{OH}$-free silica substrate [5]. From the phase behaviour, we can discard any substrate effect. As for the $F$ and $R \propto(\hbar \omega)$ spectra, they are very close to each other over the whole spectral range. In order to understand these results, we assume that, as predicted by expression (1), $\alpha$ remains roughly constant $\left(1 \mathrm{~cm}^{-1}\right)$ down to $1 \mathrm{eV}$ and then decreases exponentially with decreasing $\hbar \omega$, and that this film is covered by a thin surface layer with constant $\alpha_{s}\left(1000 \mathrm{~cm}^{-1}\right)$. Figure $2 \mathrm{~b}$ shows that the presence of such a layer may be sufficient to maintain the average absorptance level almost constant down to $0.5 \mathrm{eV}$, the measured absorption coming then essentially from the surface layer. This confirms our previous conclusions from PDS experiments performed at high modulation frequency on similar samples, in which the values of the phase for $F$ and $R$ illumination clearly indicated that most of the heat was concentrated at the film surface in the low energy range [5]. It is worth noting that the more absorbing surface layer cannot be described as a layer of "bad" a- $\mathrm{Si}: \mathrm{H}$, since its $\alpha$ values must remain roughly constant down to quite low energies. One must rather admit the existence of surface defects different from the bulk ones, either intrinsic to the surface region or induced by surface oxidation. These states should give a different DOS in the surface region, and result in a shift and pinning of the Fermi level. Band bending due to oxidation has also been suggested [14]. This would explain the observation of appreciable optical absorption in a spectral range where the bulk material has already become transparent. 

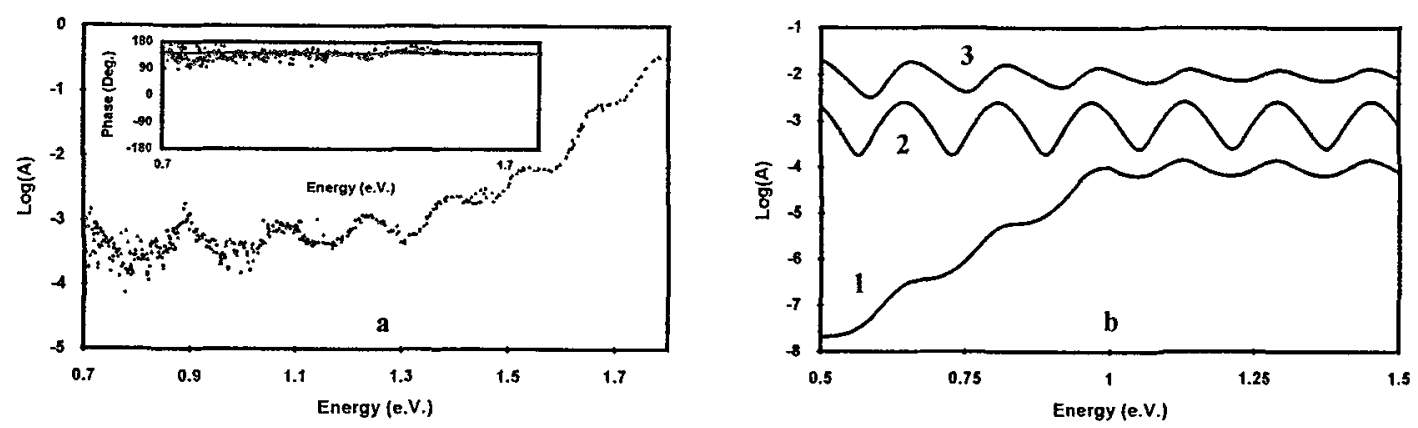

Figure 2 : (a) Calibrated experimental $A(h \omega)$ spectra obtained from PDS data with front $(\Delta)$ and rear (o) illumination for an $a-S i: H$ film $(d \simeq 1 \mu \mathrm{m}$ ) (the phase of the PDS signal is reported in the inset); (b) computed $A$ ( $\hbar \omega)$ spectra for a homogeneous film $(d=1 \mu \mathrm{m})$ with $\alpha=1 \mathrm{~cm}^{-1}$ for $\hbar \omega \geq 1 \mathrm{eV}$ and $\alpha$ decreasing from $1 \mathrm{~cm}^{-1}$ to $10^{-4} \mathrm{~cm}^{-1}$ for $0.5 \leq \hbar \omega \leq 1 \mathrm{eV}$ (1) and for a similar film (total $d=1 \mu \mathrm{m}$ ) including a surface loyer with $\alpha_{S}=10^{3} \mathrm{~cm}^{-1}$ and $d_{S}=10$ (2), 100 (3) $\mathrm{nm}$.

\section{CONCLUSION.}

We have shown by numerical simulations that PDS experiments at low modulation frequencies performed on a-Si:H thin films in their low absorption range can, in favourable cases, yield useful information on structural non-homogeneity across the film thickness resulting in variations of the optical absorption coefficient $\alpha$, but are almost insensitive to concomitant variations of the refractive index $n$, and that they may lead to serious errors in the determination of the bulk localized states density, especially for thin samples. We have also explained the unexpected behaviour of the PDS-derived $\alpha(\hbar \omega)$ spectra at low energies by the presence of an absorbing surface layer with specific defects. Such techniques based on photothermal effects are therefore complementary to ellipsometry measurements, which on the contrary are sensitive to gradients of the refractive index [15].

Acknowledgements. We thank W. Paul and his group and P. Roca i Cabarrocas for providing us with their a-Si:H samples, and for very useful discussions.

\section{REFERENCES.}

[ 1] Yasa Z.A., Jackson W.B. and Amer N.M., Appl. Opt. 21 (1982) 21.

[2] Driss-Khodja K., Gheorghiu A. and Thèye M.L., Opt. Commun. 55 (1985) 169.

[3] Buckley R.G. and Beaglehole D., Appl. Opt. 16 (1977) 2495 ; Bubenzer A. and Koidl P., Appl. Opt. 23 (1984) 2886.

[ 4] Favre M., Curtins H. and Shah A.V., J. Non-Cryst. Sol, $97 / 98$ (1987) 731.

[ 5] Chahed L., Thèye M.L., Fournier D., Roger J.P., Boccara A.C., Li Y.M., Turner W.A. and Paul W., Phys. Rev. B 43 (1991) 14488.

[6] Sládek P., Bouizem Y., Thèye M.L. and Roca i Cabarrocas P., $11^{\text {th }}$ European Photovoltaïc Solar Energy Conference, Montreux, 12-16 October 1992 (Harwood Acad. Pub. 1993) p. 710.

[7] Charbonnier F. and Fournier D., Rev. Sci. Inst. 57 (1986) 1126.

[8] Abelès F., in Advanced Optical Techniques, ed. van Heel A.C.S. (North-Holland, 1967) p. 145.

[ 9] Grillo G. and de Angelis L., J. Non-Cryst. Sol. 114 (1989) 750 ; J. Appl. Phys. 72 (1992) 3824.

[10] Amato G., Benedetto G., Boarino L. and Spagnolo R., Appl. Phys. A 50 (1990) 503 ; Sol. St. Commun. 77 (1991) 177.

[11] Asano A. and Stutzmann M., J. Non-Cryst. Sol. 137/138 (1991) 623 ; J. Appl. Phys. 70 (1991) 5025.

[12] Roca i Cabarrocas P., Bouizem Y. and Thèye M.L., Phil. Mag. B 65 (1992) 1025.

[13] Amato G., Benedetto G., Boarino L., Maringelli M. and Spagnolo R., Appl. Phys. A 52 (1991) 280.

[14] Amato G. and Fizzotti F., Phys. Rev. B 45 (1992) 14108.

[15] Parjadis de Larivière G., Frigerio J.M., Rivory J. and Abelès F., Appl. Opt. 31 (1992) 6056 ; Parjadis de Larivière G., Frigerio J.M., Bridou F. and Rivory J., Thin Solid Films 234 (1993) 458. 